ARTICLE

\title{
Predictors of venous thromboembolism among infants in children's hospitals in the United States: a retrospective Pediatric Health Information Study
}

\author{
Molly Crimmins Easterlin (iD ${ }^{1 凶}$, Yijie Li $^{2}$, Leah Yieh (iD ${ }^{2}$, Cynthia L. Gong ${ }^{2}$, Julie Jaffray ${ }^{3}$, Matt Hall ${ }^{4}$, Philippe S. Friedlich ${ }^{2}$ and \\ Ashwini Lakshmanan (iD ${ }^{2}$
}

(c) The Author(s), under exclusive licence to Springer Nature America, Inc. 2021

\begin{abstract}
OBJECTIVES: Examine: (1) Prevalence of diagnosed venous thromboembolism (VTE) in infants $<6$ months discharged from U.S. NICUs; (2) Associations between sociodemographic and clinical factors and VTE; (3) Secondary outcomes related to VTE. STUDY DESIGN: Multivariable logistic regressions examined associations between VTE and sociodemographic and clinical factors among infants <6 months discharged from Pediatric Health Information System (PHIS) NICUs between 2016 and 2019. RESULTS: Of 201,033 infants, 2720 (1.35\%) had diagnosed VTE. Birthweight 300-1000 g (aOR 3.14, 95\% Cl 2.54-3.88), 1000-1500 g (aOR 1.77, 95\% Cl 1.40-2.42) versus $2500-3999 \mathrm{~g}$, and public (aOR 1.18, 95\% Cl 1.02-1.37) versus private insurance were associated with increased odds of VTE, as were CVC, TPN, mechanical ventilation, infection, ECMO, and surgery. All types of central lines (nontunneled and tunneled CVCs, PICCs, and umbilical catheters) had higher odds of VTE than not having that type of line. CVCs in upper versus lower extremities had higher odds of VTE.

CONCLUSION: Infants with risk factors may require monitoring for VTE. Results may inform VTE prevention.
\end{abstract}

Journal of Perinatology (2022) 42:103-109; https://doi.org/10.1038/s41372-021-01232-1

\section{INTRODUCTION}

Studies of large nationally representative databases have shown increasing rates of venous thromboembolism (VTE) among neonates and infants. Between 2001 and 2007, the annual rate of VTE increased by $70 \%$ in infants $<28$ days and $100 \%$ in infants 28 days to 1 year among patients in the Pediatric Health Information System (PHIS) [1]. Similarly, the rate of VTE increased from 18.1 per 100,000 in 1994 to 49.6 per 100,000 in 2009 among infants in the National Inpatient Sample [2]. Though not definitively established, the increasing rate of VTE among infants may be partially due to increased survival among the most premature and sickest infants, increased use of central venous catheters (CVCs), and increased screening and diagnosis $[1,3,4]$.

The increasing rate of neonatal VTE is concerning since VTE has been associated with increased short-term morbidity (clot progression, pulmonary embolism, and stroke), long-term morbidity (recurrent VTE and post-thrombotic syndrome), as well as mortality, increased length of stay, and higher healthcare costs [3]. In neonates specifically, VTE associated with a CVC may necessitate the removal of the line and treatment with anticoagulation. This may then increase the number of central access procedures and vascular injury as well as increase the risk of bleeding, especially in premature infants already at risk of intraventricular hemorrhage.
Despite the increasing rates of neonatal VTE and its association with worse outcomes, no standards or protocols exist for screening, prevention, diagnosis, or treatment. This is in contrast to adult patients where risk factors for VTE are relatively well understood and have led to prevention strategies [5-8]. The absence of neonatal VTE protocols is partially due to a lack of understanding of risk factors for neonatal VTE, especially given their immature coagulation system [9-12], relatively small vessel diameters, critical disease in much of the tertiary and quaternary units $[3,4]$, relatively small sample sizes in past studies, and the complexity and variability of the neonatal intensive care unit (NICU) population.

Initial neonatal VTE data came from prospective registries in Canada, Germany, and Denmark in the 1990s [13-15]. These studies were instrumental to beginning to understand neonatal VTE and identified initial risk factors, with CVCs being the greatest. Given the relatively rare occurrence of neonatal VTE, the numbers of patients who had the outcome of interest was low, with thrombosis occurring in $<100$ patients in these studies. These were followed by retrospective case series and case-control studies, which continued to identify possible risk factors (CVC, infection, prematurity/small for gestational age, maternal diabetes, and dehydration/polycythemia) [4, 16-18] but were also limited by small sample sizes, subpopulations (congenital heart disease),

\footnotetext{
${ }^{1}$ Division of Neonatology, Department of Pediatrics, LAC + USC Medical Center, Keck School of Medicine, University of Southern California, Los Angeles, CA, USA. ${ }^{2}$ Fetal and Neonatal Institute, Division of Neonatology, Children's Hospital Los Angeles, Department of Pediatrics, Keck School of Medicine, University of Southern California, Los Angeles, CA, USA. ${ }^{3}$ Cancer and Blood Disease Institute, Children's Hospital Los Angeles, Department of Hematology, Oncology and Blood and Marrow Transplantation, Keck School of

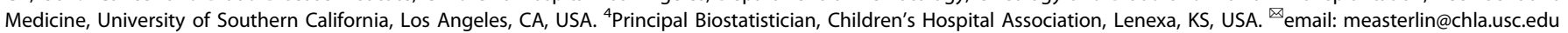


specific type of central lines (umbilical venous catheters, peripherally inserted central catheters (PICCs)), or risk factors (infection) [19-21]. Additionally, more recent data indicate the incidence of neonatal VTE is now higher than was reported in the 1990 s and early 2000s [4, 22]. Since that time, the NICU population and NICU care has changed-encompassing younger, smaller, more complex, and sicker patients, with likely increased use of CVCs, and advances in life-sustaining measures, such as ventilator modalities and total parenteral nutrition (TPN) composition. Other reasons for the increased incidence of VTE include increased awareness of VTE within the medical community and improved imaging techniques. As such, there is a need for updated data and a more encompassing approach to studying risk factors among larger numbers of patients in order to inform the development of prevention protocols.

Therefore, we sought to examine neonatal VTE using a large nationally representative database of free-standing children's hospitals to: (1) examine the current prevalence of diagnosed VTE in infants $<6$ months, (2) evaluate associations between sociodemographic factors and VTE and clinical factors, such as CVCs, and VTE, and (3) assess secondary outcomes related to VTE.

\section{METHODS \\ Study design and data}

We performed a retrospective cross-sectional analysis of data from the Pediatric Health Information System (PHIS). PHIS is an administrative discharge database that includes clinical and resource utilization data for inpatient encounters from about 50 (depending on the year) children's hospitals, including demographic data, clinical details of the inpatient encounter, diagnosis codes, and procedure codes [23]. The data are deidentified and undergo multiple reliability and validity checks. The institutional review board of Children's Hospital Los Angeles approved this study as exempt (CHLA-20-00182).

\section{Study sample}

We included all infants discharged from PHIS hospital NICUs between January 1, 2016 and December 31, 2019 who were $<6$ months of age at discharge. January 1, 2016 was selected as the earliest discharge date to include as the transition from the prior coding system for healthcare transactions such as medical diagnosis and procedures (International Classification of Diseases-9 (ICD-9)) to the new coding system (ICD-10), which was mandated by the Department of Health and Human Services to start on October 1, 2015, should have been well established by that time [24]. The analysis was limited to infants $<6$ months of age at the time of discharge due to the developmental hemostatic changes that occur after that age [25]. Readmissions were excluded given the difficulty in attributing outcomes to occurrences in the current admission versus previous admission. Fifty PHIS NICUs reported relevant data and were therefore included in our analysis.

\section{Outcomes}

The outcome of interest, VTE, was identified using ICD-10 Clinical Modification (ICD-10-CM) diagnosis codes. VTE included thrombosis and embolism (acute and chronic) or phlebitis and thrombophlebitis, in the upper extremities (superficial and deep), portal vein, renal vein, vena cava, other thoracic veins, lower extremities (superficial and deep), and other or unspecified veins, as well as thrombosis or embolism due to a vascular device. These VTEs were therefore "diagnosed" VTEs, as it was predicated on the patient having been assigned the ICD-10 CM diagnosis code. Phlebitis, thrombophlebitis and superficial vessels were included due to often being investigated in a similar way to VTE (ultrasound) and may be managed similarly (line removal). Supplementary Table 1 provides the details on ICD-10 diagnosis codes used to identify VTE and their relative prevalence. Secondary outcomes examined were length of stay and mortality.

\section{Independent variables}

Independent variables examined include sociodemographic factors and clinical factors thought to potentially be risk factors for VTE either based on previous literature or Virchow's triad. Sociodemographic factors included birthweight, sex, race, ethnicity, health insurance, and hospital region. Clinical factors included receipt of TPN, mechanical ventilation, infection, need for extracorporeal membrane oxygenation (ECMO), surgery, and presence of a CVC. We also examined different types of CVCs (tunneled and non-tunneled CVCs vs. PICCs vs. umbilical catheters) and different locations of CVC insertion (upper extremity versus lower extremity). The clinical factors (other than presence of CVC) are identified by PHIS using ICD-10 or clinical transaction codes to determine whether the patient had (infection) or received (TPN, mechanical ventilation, ECMO) that clinical item during the hospitalization. The presence of a CVC was determined using a combination of ICD-10 procedure codes to identify a CVC terminating in a central location according to the Society of Critical Care Medicine [26], ICD-10 diagnosis codes, PHIS billing codes, and Current Procedural Terminology (CPT) codes (Supplementary Table 2). PHIS billing codes, CPT codes, and ICD-10 procedure codes were used to determine line type. ICD-10 procedure codes were used to determine upper or lower extremity insertion site, the location of the central line tip, and the surgical approach (open, percutaneous, or percutaneous endoscopic).

\section{Statistical analysis}

Univariate analyses examined distributions of the independent variables, outcome, and secondary outcomes. Bivariate analyses included chi-square tests for categorical variables and $t$ tests for continuous variables. Separate multivariable logistic regressions examined associations between (1) VTE and sociodemographic factors, (2) VTE and clinical factors, and (3) VTE and line type or upper extremity versus lower extremity line insertion site. All statistical analyses were performed in Stata, version SE 16.1 (StataCorp). To adjust for clustering at the hospital level the robust and cluster options were used. Code is available by request from the corresponding author.

\section{RESULTS}

\section{Analytic sample}

The analysis included 201,033 infants $<6$ months discharged from PHIS NICUs between 2016 and 2019. Mean (standard deviation, SD) gestational age was 35.64 (4.16) weeks and mean (SD) birthweight was 2686.51 (942.20) g (Table 1). Slightly over half (55.9\%) were male and white $(55.1 \%)$ and the majority were nonHispanic $(72.5 \%)$. More infants had public $(56.0 \%)$ versus private (41.0\%) insurance. Hospitals were relatively evenly distributed throughout the country with the northeast being slightly less represented.

\section{Prevalence of VTE}

The prevalence of diagnosed VTE in infants $<6$ months of age discharged from PHIS NICUs was $1.35 \%(2,720 / 201,033)$. Both the numerator and denominator are inclusive of infants who died, as such a case would be discharged as a mortality. There was significant variability in the prevalence of VTE among hospitals (Fig. 1), with the prevalence ranging from 0.1 to $5.9 \%$. VTE was most commonly coded as being in the lower extremities (35.0\%), associated with vascular devices (29.7\%), or in the vena cava and other thoracic veins (26.1\%) (Table 2 ).

In bivariate analyses, infants who had a VTE were more likely to be of lower gestational age (mean (SD) 34.19 (5.14) versus 35.66 (4.14) weeks, $p<0.0001$ ) and lower birthweight (mean (SD) 2392.21 (1072.50) versus 2690.37 (939.76) $g, p<0.0001$ ) than those who did not have a VTE (Table 1). They were also more likely to be Hispanic (19.23\% versus $16.01 \%)$, have public insurance (60.92\% versus $55.97 \%$ ), and be cared for in a hospital located in the West $(24.12 \%$ versus $20.73 \%)$. In terms of clinical factors, in bivariate analysis, infants who had a VTE were more likely to have received mechanical ventilation, TPN, had an infection, been on ECMO, and had surgery compared to infants who did not have a VTE. Eighty-five percent of infants with a VTE had a CVC versus $24.6 \%$ of infants without a VTE. The prevalence of VTE among those with a CVC was $3.78 \%$ compared to $0.32 \%(p<0.001)$ in those without a central line.

In those without a VTE, the principal diagnoses reflect mode of delivery and common NICU problems, including respiratory 
Table 1. Descriptive statistics for the entire sample, infants with venous thromboembolism (VTE), and infants without venous thromboembolism.

\begin{tabular}{|c|c|c|c|c|}
\hline & $\begin{array}{l}\text { Entire sample } \\
(N=201,033)\end{array}$ & $\begin{array}{l}\text { NICU Admissions without VTE } \\
(N=198,313)\end{array}$ & $\begin{array}{l}\text { NICU Admissions with VTE } \\
(N=2,720)\end{array}$ & \\
\hline $\begin{array}{l}\text { Gestational age (completed weeks) } \\
(N=161,282)\end{array}$ & $35.64(4.16)$ & $35.66(4.14)$ & $34.19(5.14)$ & $<0.0001$ \\
\hline $22-25$ & $5691(3.53)$ & $5479(3.44)$ & $212(9.87)$ & $<0.001$ \\
\hline $26-31$ & $19,046(11.81)$ & $18,656(11.72)$ & $390(18.16)$ & \\
\hline $32-33$ & $13,613(8.44)$ & $13,475(8.47)$ & $138(6.43)$ & \\
\hline $37-40$ & $79,836(49.50)$ & $78,914(49.59)$ & $922(42.94)$ & \\
\hline $41-44$ & 5940 (3.68) & $5888(3.70)$ & $52(2.42)$ & \\
\hline $\begin{array}{l}\text { Birthweight (grams) } \\
(N=183,335)\end{array}$ & $2686.51(942.20)$ & 2690.37 (939.76) & $2392.21(1072.50)$ & $<0.0001$ \\
\hline 300-999 & $11,223(6.12)$ & $10,920(6.00)$ & $303(15.49)$ & $<0.001$ \\
\hline $\begin{array}{l}\text { Sex } \\
(N=200,826)\end{array}$ & & & & 0.385 \\
\hline Male & $112,209(55.87)$ & $110,668(55.86)$ & $1541(56.70)$ & \\
\hline Female & $88,617(44.13)$ & $87,440(44.14)$ & $1177(43.30)$ & \\
\hline Race $(201,033)$ & & & & 0.001 \\
\hline Black & 35,465 (17.64) & $34,969(17.63)$ & $496(18.24)$ & \\
\hline Asian/American Indian/Pacific Islander & $10,229(5.09)$ & $10,091(5.09)$ & $138(5.07)$ & \\
\hline White & $110,733(55.08)$ & $109,198(55.06)$ & $1535(56.43)$ & \\
\hline Other & $24,623(12.25)$ & $24,277(12.24)$ & $346(12.72)$ & \\
\hline Unknown & $19,983(9.94)$ & $19,778(9.97)$ & $205(7.54)$ & \\
\hline Private & $81,239(40.95)$ & $80,252(41.00)$ & 987 (37.09) & \\
\hline Public & $111,181(56.04)$ & $109,560(55.97)$ & $1621(60.92)$ & \\
\hline Other & $5979(3.01)$ & $5926(3.03)$ & $53(1.99)$ & \\
\hline \multicolumn{5}{|l|}{ Clinical factors } \\
\hline $\begin{array}{l}\text { Mechanical ventilation } \\
(N=201,033)\end{array}$ & $69,496(34.57)$ & $67,190(33.88)$ & $2306(84.78)$ & $<0.001$ \\
\hline $\begin{array}{l}\text { TPN } \\
(N=201,033)\end{array}$ & $77,282(38.44)$ & 74,845 (37.74) & $2437(89.60)$ & $<0.001$ \\
\hline $\begin{array}{l}\text { Infection } \\
(N=201,033)\end{array}$ & $45,001(22.38)$ & $43,312(21.84)$ & $1689(62.10)$ & $<0.001$ \\
\hline $\begin{array}{l}\text { ECMO } \\
(N=201,033)\end{array}$ & $1875(0.93)$ & $1601(0.81)$ & $274(10.07)$ & $<0.001$ \\
\hline $\begin{array}{l}\text { Surgery } \\
(N=201,033)\end{array}$ & $34,771(17.30)$ & $33,061(16.67)$ & $1710(62.87)$ & $<0.001$ \\
\hline $\begin{array}{l}\text { Central Venous Catheter } \\
(N=193,112)\end{array}$ & & & & $<0.001$ \\
\hline No & $144,009(74.57)$ & $143,597(75.42)$ & $412(15.19)$ & \\
\hline Yes & $49,103(25.43)$ & $46,803(24.58)$ & $2300(84.81)$ & \\
\hline \multicolumn{5}{|l|}{ Hospital level characteristics } \\
\hline Census Region $(201,033)$ & & & & $<0.001$ \\
\hline Midwest & $61,079(30.38)$ & $60,390(30.45)$ & $689(25.33)$ & \\
\hline
\end{tabular}


Table 1 continued

\begin{tabular}{|c|c|c|c|c|}
\hline & $\begin{array}{l}\text { Entire sample } \\
(N=201,033)\end{array}$ & $\begin{array}{l}\text { NICU Admissions without VTE } \\
(N=198,313)\end{array}$ & $\begin{array}{l}\text { NICU Admissions with VTE } \\
(N=\mathbf{2}, 720)\end{array}$ & \\
\hline Northeast & $27,869(13.86)$ & $27,502(13.87)$ & $367(13.49)$ & \\
\hline West & $41,767(20.78)$ & $41,111(20.73)$ & $656(24.12)$ & \\
\hline \multicolumn{5}{|c|}{ Secondary outcomes } \\
\hline $\begin{array}{l}\text { Length of Stay } \\
(N=201,033)\end{array}$ & $21.01(29.09)$ & $20.41(28.31)$ & $64.66(46.38)$ & $<0.0001$ \\
\hline
\end{tabular}

TPN total parenteral nutrition, ECMO extracorporeal membrane oxygenation.

Includes all NICU discharges $<6$ months of age from PHIS Hospitals between Jan 1, 206 and Dec 31, 2019. Total N=201,033. 2,720 with VTE (1.35\%). T-test used for continuous variables, chi-square for categorical variables.

Missingness: birthweight $8.80 \%$, sex $0.10 \%$, insurance $1.31 \%$, central venous catheter $3.94 \%$, and gestational age $19.77 \%$ (gestational age was not included in regression analysis due to collinearity with birthweight and high percentage of missingness).

Race Other identifies a patient's race as "Other".

Private insurance includes HMO/PPO; Public insurance includes Medicaid, Medicare, military, CHIP; Other insurance includes self-pay, charity, other payor, hospital did not bill.

Mechanical ventilation identifies if a patient received a mechanical vent during this episode of care. $Y=$ Patient had an ICD-9 Px code of $96.70,96.71$ or 96.72 , ICD-10 Px code of 5A1935Z, 5A1945Z or 5A1955Z or a charge mapped to CTC code 521166 or 521169 . N = No mechanical vent. (See PHIS website).

TPN identifies if a patient received TPN (total parenteral nutrition) during this episode of care. $Y=$ Patient had a charge mapped to any of the following CTC codes: $146011,146040,146041,146070 . \mathrm{N}=$ No TPN charges. (See PHIS website).

Infection identifies an infection as defined by the ICD codes recorded that trigger this flag. $\mathrm{Y}=$ Patient had a diagnosis that was considered an infection. $\mathrm{N}=$ No infection. (See PHIS website).

ECMO identifies if a patient received ECMO (extracorporeal membrane oxygenation) during this episode of care. $Y=$ Patient had an ICD-9 Px code of 39.65 , ICD-10 Px code of 5A15223 or a charge mapped to CTC code 521180, 521181 and 521182. N = No ECMO (See PHIS website).

Surgery identifies if a patient was billed for Operating Room services during this episode of care. $\mathrm{Y}=$ Patient had a charge mapped to a CTC code of 611110 . $\mathrm{N}=$ No Operating Room charges. (See PHIS website).

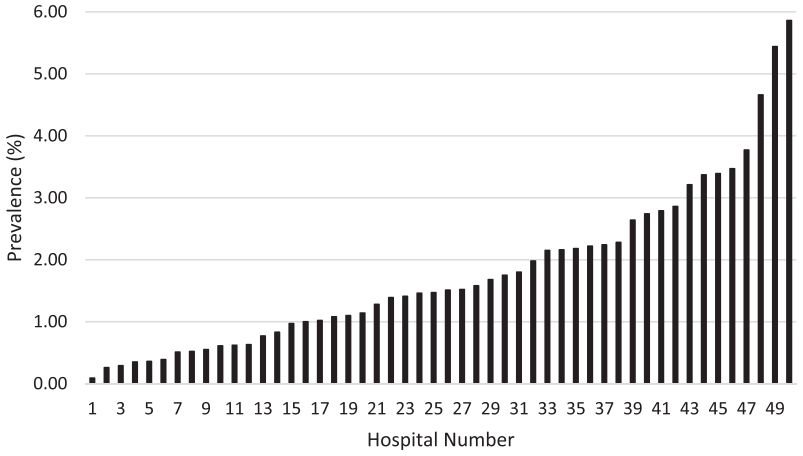

Fig. 1 Prevalence of venous thromboembolism by hospital. Includes 50 PHIS hospitals. Prevalence range $0.09-5.86 \%$.

Table 2. Most common locations and factors associated with VTE.

\begin{tabular}{|lll|}
\hline Associated factors & Frequency & Percent \\
\hline Lower extremity & 953 & 35.04 \\
\hline Due to vascular device & 807 & 29.67 \\
\hline Vena cava and other thoracic veins & 711 & 26.14 \\
\hline Upper extremity & 476 & 17.50 \\
\hline Portal Vein/Budd Chiari & 391 & 14.38 \\
\hline Phlebitis and thrombophlebitis & 371 & 13.64 \\
\hline Other and unspecified veins & 166 & 6.10 \\
\hline
\end{tabular}

$N=2720$. Displays summary percentages for VTE in different areas of body (based in ICD-10 Diagnosis Codes). For VTE acute and chronic embolism and thrombosis were combined. distress or failure, low birthweight, jaundice, and hypoglycemia. In comparison, the principal diagnosis in infants with a VTE tends to reflect a more medically complex population-with gastroschisis, congenital heart disease, and sepsis falling in the top 10 most common principal diagnoses. Supplementary Table 3 compares the ten most common principal diagnoses in infants without a VTE versus those with VTE.

\section{Prevalence and location of central venous catheters}

Hospitals bill (or in some cases may not bill), and therefore code, for CVC insertion in varying ways. Using a combination of ICD-10 procedure codes, ICD-10 diagnosis codes, PHIS clinical transaction procedure codes, and CPT codes, we sought to ascertain whether the infant had a CVC. Using this system, information was available on $96 \%$ of our sample (Supplementary Table 4). Umbilical lines, PICC lines, and non-tunneled and tunneled CVCs were more common in those who had a VTE than those who did not $(23.27 \%$ vs. $10.18 \%$ for umbilical lines, $41.49 \%$ vs. $8.90 \%$ for PICC lines, $12.65 \%$ vs. $1.67 \%$ for non-tunneled and tunneled CVCs, respectively). The most common locations of the line tip were superior vena cava, followed by inferior vena cava (IVC), then IVC via an umbilical approach, then right atrium, left subclavian, and right subclavian (Supplementary Table 4). The most common approach by far was percutaneous.

\section{Sociodemographic risk factors for VTE}

In multivariable regression examining sociodemographic risk factors for VTE, extremely low birthweight $<1000$ grams (aOR, 3.14; $95 \% \mathrm{Cl}$, 2.54-3.88) and very low birthweight birthweight 1000 to 1500 grams (aOR, 1.77; 95\% Cl, 1.40-2.24) were associated with a higher odds of VTE compared to birthweight 2500 to $3999 \mathrm{~g}$ (Fig. 2). Public insurance (aOR, 1.18; 95\% Cl, 1.02-1.37) versus private insurance was associated with a higher odds of VTE. None of the factors were associated with a significantly decreased risk of VTE. 


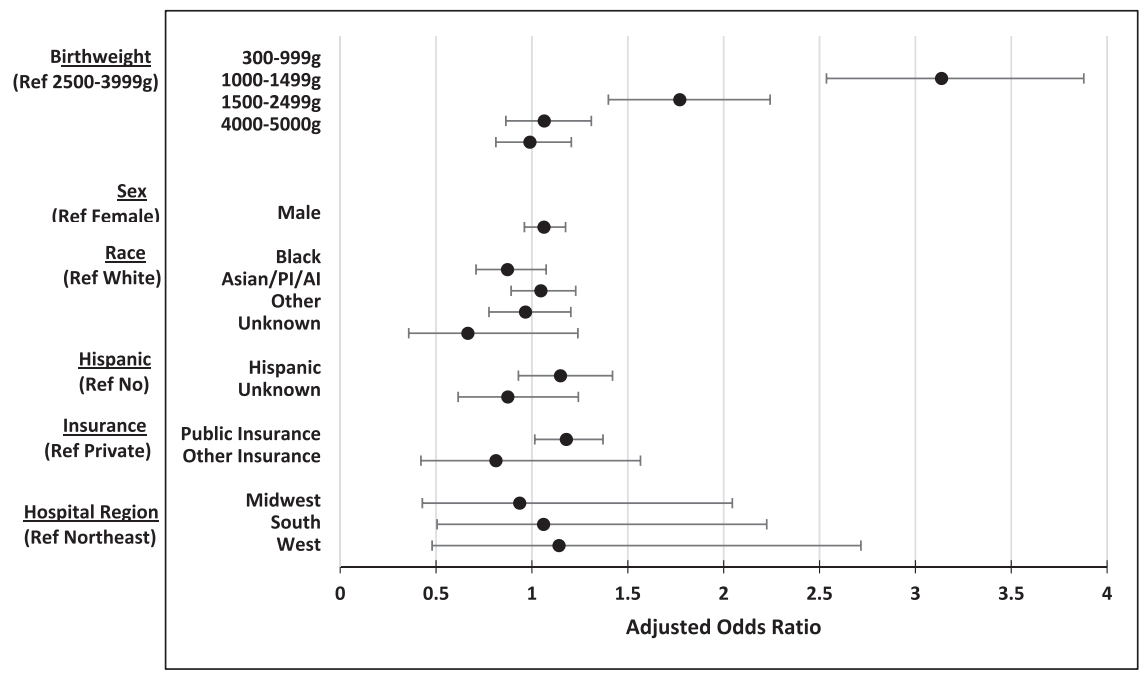

Fig. 2 Multivariable regression of sociodemographic risk factors on venous thromboembolism. Adjusted odds ratio and $95 \%$ confidence interval displayed. $N=180,870$. Race Other identifies a patient's race as "Other" than White, Black, Asian/Pacific Islander/American Indian, or Unknown. Private insurance includes HMO/PPO; Public insurance includes Medicaid, Medicare, military, CHIP; Other insurance includes selfpay, charity, other payor, hospital did not bill.

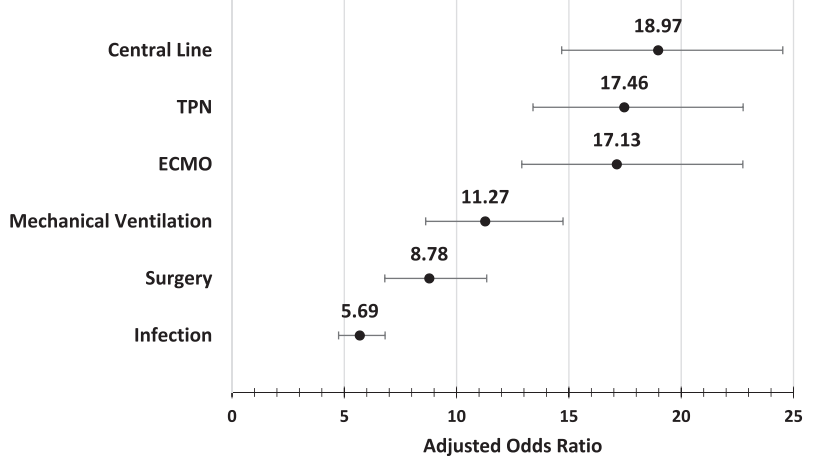

Fig. 3 Multivariable regression of clinical risk factors on venous thromboembolism. Adjusted odds ratio and $95 \%$ confidence interval displayed. Adjusted for birthweight, sex, race, Hispanic ethnicity, insurance, and hospital region. $N=180,870$ for all clinical factors except central venous catheter where $N=173,733$. TPN total parenteral nutrition, ECMO extracorporeal membrane oxygenation.

\section{Clinical risk factors for VTE}

In multivariable regression examining clinical risk factors for VTE while controlling for sociodemographic risk factors, all six clinical risk factors examined-any central line (aOR, 18.97; 95\% Cl, 14.68-24.52), TPN (aOR, 17.46; 95\% Cl, 13.40-22.76), ECMO (aOR, 17.13; 95\% Cl 12.90-22.75), mechanical ventilation (aOR, 11.27; $95 \% \mathrm{Cl}, 8.62-14.74)$, surgery (aOR, 8.78; 95\% Cl, 6.80-11.34), and infection (aOR, 5.69; 95\% Cl, 4.75-6.82)—were significantly associated with an increased odds of VTE (Fig. 3).

\section{Central line type and insertion site and VTE}

We also examined how central line type and location of insertion were associated with risk of VTE. After adjusting for sociodemographic factors, all types of central lines were associated with a higher odds of VTE than not having that type of central line - non-tunneled and tunneled CVC versus no CVC, aOR, 8.50; $95 \% \mathrm{Cl}(5.20-13.90)$, umbilical line versus no umbilical line, aOR, 2.32; 95\% Cl (1.82-2.95), and PICC versus no PICC, aOR, 6.93; 95\% (Cl 5.55-8.65). Central lines inserted in the upper extremities were associated with a higher odds of VTE (versus no central line, aOR, $8.43,95 \% \mathrm{Cl} 6.64-10.70$ ) than lines inserted in veins of the lower extremity (versus no line, aOR 5.49, 95\% Cl 4.33-6.95); however, having both an upper and lower extremity line was associated with the highest odds of VTE (versus no line, aOR $16.18,95 \% \mathrm{Cl}$ 12.66-20.68).

\section{Secondary outcomes}

VTE was significantly associated with increased mortality $(13.4 \%$ in those with VTE vs. 3.5\% in those without VTE, $p<0.001)(a O R, 3.36$; $95 \% \mathrm{Cl} 2.57-4.40)$ and length of stay [mean (SD), 64.66 (46.33) vs. 20.50 (28.43) days, $p<0.001$ ] (coeff, 38.25; 95\% Cl 35.43-41.07).

\section{DISCUSSION}

In this analysis of a large administrative database, the prevalence of diagnosed VTE was $1.35 \%$ in those $<6$ months of age, or $13.5 / 1000$ NICU discharges, and the prevalence varied widely among PHIS NICUs. We identified significant associations between some demographic factors and VTE, including low birthweight and public insurance, and significant associations between some clinical factors and VTE, including any CVC, TPN, mechanical ventilation, infection, $E C M O$, and surgery. We also found that all types of central lines (non-tunneled and tunneled CVCs, PICCs, and umbilical catheters) were associated with a higher odds of VTE than not having that type of line, and upper extremity CVCs were associated with a higher odds of VTE than lower extremity CVCs. VTE was associated with significantly increased mortality and longer length of stay, which may be because these infants also tend to be more medically complex or sicker.

The prevalence of diagnosed VTE that we found is higher than the initial incidence reported from the late 1990s to early 2000s in the German registry among neonates $\leq 4$ weeks $(5.1 / 100,000$ live births) [14] and Dutch registry (14.5/10,000 children 0-28 days and $0.25 / 10,000$ children $29-265$ days), which may be due to changes in patient population and clinical care since that time [15]. Though we acknowledge that prevalence and incidence are not equivalent, that the prevalence we measure is per discharged NICU patients (not per live births or per NICU admissions), and that the age ranges vary by study, this is included for historical context. Our increased prevalence is supported by a similarly increased incidence found more recently in a clinical database $(15 / 1000$ NICU admissions) [4]. Our finding of wide variability in prevalence of neonatal VTE among NICUs has not been previously reported. It is possible that some of this variability may be due to differences in screening for and diagnosis of VTE as well as differences in 
coding and/or billing. Case-mix may also be part of the variability, however, given that PHIS includes level 3 and level 4 NICUs only, these institutions should see relatively similar patient populations. Given the magnitude of differential, and variation known to exist in other aspects of care (i.e., infection), the hospital variation in neonatal VTE prevalence deserves further investigation. In fact, studies have shown variability in central line practices among NICUs, such as in aspects of PICC insertion and maintenance [27].

In terms of risk factors for VTE, other studies have also identified CVC $[2,13,14,18,28,29]$, infection [13, 14, 21, 29], and mechanical ventilation $[2,29]$ to be risk factors; our study supports these and further adds to the list of potential risk factors by identifying significant associations. It also expands our understanding of the types of central lines and locations of insertion that are associated with higher odds of VTE, as other studies have examined complications of PICCs by upper or lower extremity location and found conflicting results. A meta-analysis of eight studies showed that upper extremity PICCs were associated with a lower risk of thrombosis compared to lower extremity PICCs [30], while two other studies found no difference in thrombosis in upper versus lower extremity PICCs [31, 32]. These studies differ from ours in that they are restricted to PICC lines and have much smaller sample sizes, which can be problematic when studying a low frequency outcome. However, while we only sought to examine VTE, these other studies examine multiple complications and make clear that it will be necessary to balance various other complications of central lines in addition to VTE when constructing a prevention protocol.

\section{Limitations}

This study is limited by the nature of the administrative discharge database, which relies on coding and billing and can sometimes lack granularity and have missing data. However, PHIS undergoes multiple reliability and validity checks. In our study, other than birthweight $(8.80 \%)$, missingness was generally $<5 \%$ (see Table 1 Notes). It is also limited by the reliance on ICD-10 codes to identify the outcome. In a study of ICD-10 codes for venous and arterial thrombosis in the Danish National Patient Registry, the positive predictive value among admitted children 0-18 years was $77.4 \%$ among neonates ( $<28$ days) and $51.2 \%$ in children 28 days 14 years [33]. We capture VTEs that are "diagnosed" by being assigned an ICD-10 code, but the true prevalence of VTEs (including those that are undiagnosed and not coded) is unknown and cannot be estimated. Another limitation is that how VTEs were "diagnosed", such as with imaging or clinical symptoms, is unknown. Additionally, our findings are specific to the PHIS NICU population. The generalizability to non-PHIS hospitals may be limited. The PHIS sample includes only free-standing children's hospitals and VTE is seen more commonly in children's hospitals [34]. Finally, as with all retrospective studies, this study cannot prove causality but only establish associations that deserve further investigation.

There are also limitations in using the administrative database to analyze central lines [35]. There may be systematic errors in the coding of central lines as hospitals bill for these differently. It is reassuring that about $96 \%$ of our sample had information using our approach. Additionally, the temporal relationship between line placement and clot development is difficult to establish and was not examined in this study. Similarly, patients may have had multiple central lines and attributing a clot to a specific line was not possible. Due to design of the PHIS database, we were unable to evaluate the temporal relationship between anticoagulation, VTE and a CVC. We were also unable to distinguish asymptomatic catheter associated thrombi which resolved with catheter removal alone. In the future, we hope to further address some of these limitations by including timing of line placement and clot development, and number of line days. It is likely that hospital policies and practices, in addition to patient factors, play a role in risk of neonatal VTE

The importance of this study is that while overall VTE risk is low, it is rising. Consistent with other studies, we identified a population that is especially at risk: the sickest and smallest NICU patient. Therefore, our study is hypothesis generating, identifying factors that should be further examined with a multi-institutional prospective data collection, which could ultimately help lead to development of a risk stratification index and VTE prevention and surveillance protocol. A multi-institutional registry of pediatric hospital-acquired thrombosis cases has been established and the development of a risk stratification tool for pediatric patients is underway [3]. This tool will need to be modified for the neonatal population and our study has provided some insight into potential modifications and areas for future research.

\section{CONCLUSIONS}

In conclusion, in this analysis of diagnosed VTE in children's hospitals in the U.S., we found the prevalence was $1.35 \%$ among infants $<6$ months discharged from PHIS NICUs but varied among centers. We identified significant associations between demographic factors (low birthweight, public insurance) and VTE and as well as clinical factors (TPN, mechanical ventilation, infection, ECMO, surgery, any central line, and upper extremity lines) and VTE. Additionally, VTE was associated with significantly increased odds of mortality and longer length of stay. In the future, we hope to expand understanding of VTE, by examining work up of suspected or confirmed VTE (including imaging and laboratory studies undertaken), treatment with anticoagulation, and associated costs of care.

\section{REFERENCES}

1. Raffini L, Huang YS, Witmer C, Feudtner C. Dramatic increase in venous thromboembolism in children's hospitals in the United States from 2001 to 2007. Pediatrics. 2009;124:1001-8. https://doi.org/10.1542/peds.2009-0768.

2. Boulet SL, Grosse SD, Thornburg CD, Yusuf H, Tsai J, Hooper WC. Trends in venous thromboembolism-related hospitalizations, 1994-2009. Pediatrics. 2012;130: e812-20. https://doi.org/10.1542/peds.2012-0267.

3. Jaffray J, Mahajerin A, Young G, Goldenberg N, Ji L, Sposto R, et al. A multiinstitutional registry of pediatric hospital-acquired thrombosis cases: the Children's Hospital-Acquired Thrombosis (CHAT) project. Thromb Res. 2018;161:67-72. https://doi.org/10.1016/j.thromres.2017.11.019.

4. Bhat R, Kumar R, Kwon S, Murthy K, Liem RI. Risk factors for neonatal venous and arterial thromboembolism in the neonatal intensive care Unit-A case control study. J Pediatr. 2018;195:28-32. https://doi.org/10.1016/j.jpeds.2017.12.015.

5. Geerts WH, Bergqvist D, Pineo GF, Heit JA, Samama CM, Lassen MR, et al. Prevention of venous thromboembolism: American College of Chest Physicians Evidence-Based Clinical Practice Guidelines (8th Edition). Chest. 2008;133:381S-453S. https://doi.org/ 10.1378/chest.08-0656.

6. Rocha AT, Paiva EF, Lichtenstein A, Milani R Jr, Cavalheiro CF, Maffei FH. Riskassessment algorithm and recommendations for venous thromboembolism prophylaxis in medical patients. Vasc Health Risk. 2007;3:533-53.

7. Woller SC, Stevens SM, Jones JP, Lloyd JF, Evans RS, Aston VT, et al. Derivation and validation of a simple model to identify venous thromboembolism risk in medical patients. Am J Med. 2011;124:947-54.e2. https://doi.org/10.1016/j. amjmed.2011.06.004

8. Decousus H, Tapson VF, Bergmann JF, Chong BH, Froehlich JB, Kakkar AK, et al. Factors at admission associated with bleeding risk in medical patients: findings from the IMPROVE investigators. Chest. 2011;139:69-79. https://doi.org/10.1378/ chest.09-3081.

9. Andrew M, Paes B, Milner R, Johnston M, Mitchell L, Tollefsen DM, et al. Development of the human coagulation system in the full-term infant. Blood. 1987;70:165-72.

10. Andrew M, Paes B, Milner R, Johnston M, Mitchell L, Tollefsen DM, et al. Development of the human coagulation system in the healthy premature infant. Blood. 1988;72:1651-7.

11. Andrew $M$, Paes $B$, Johnston $M$. Development of the hemostatic system in the neonate and young infant. Am J Pediatr Hematol Oncol. 1990;12:95-104. https:// doi.org/10.1097/00043426-199021000-00019.

12. Achey MA, Nag UP, Robinson VL, Reed CR, Arepally GM, Levy JH, et al. The developing balance of thrombosis and hemorrhage in pediatric surgery: clinical implications of age-related changes in hemostasis. Clin Appl Thromb Hemost. 2020;26:1076029620929092. https://doi.org/10.1177/1076029620929092. 
13. Schmidt B, Andrew M. Neonatal thrombosis: report of a prospective Canadian and international registry. Pediatrics. 1995;96:939-43.

14. Nowak-Göttl U, von Kries R, Göbel U. Neonatal symptomatic thromboembolism in Germany: two year survey. Arch Dis Child Fetal Neonatal Ed. 1997;76:F163-7. https://doi.org/10.1136/fn.76.3.f163.

15. van Ommen $\mathrm{CH}$, Heijboer $\mathrm{H}$, Büller HR, Hirasing RA, Heijmans HS, Peters M. Venous thromboembolism in childhood: a prospective two-year registry in The Netherlands. J Pediatr. 2001;139:676-81. https://doi.org/10.1067/mpd.2001.118192.

16. Unal S, Gönülal D, Siyah Bilgin B, Koşan Çulha V, Yarali N. Experience and prognosis of systemic neonatal thrombosis at a level III NICU. J Pediatr Hematol Oncol. 2018;40:e410-4. https://doi.org/10.1097/MPH.0000000000001218.

17. Sirachainan $N$, Limrungsikul A, Chuansumrit A, Nuntnarumit $P$, Thampratankul $L$, Wangruangsathit $S$, et al. Incidences, risk factors and outcomes of neonatal thromboembolism. J Matern Fetal Neonatal Med. 2018;31:347-51. https://doi. org/10.1080/14767058.2017.1285892.

18. Amankwah EK, Atchison CM, Arlikar S, Ayala I, Barrett L, Branchford BR, et al. Risk factors for hospital-asssociated venous thromboembolism in the neonatal intensive care unit. Thromb Res. 2014;134:305-9. https://doi.org/10.1016/j.thromres.2014.05.036.

19. Faraoni D, Gardella KM, Odegard KC, Emani SM, DiNardo JA. Incidence and predictors for postoperative thrombotic complications in children with surgical and nonsurgical heart disease. Ann Thorac Surg. 2016;102:1360-7. https://doi. org/10.1016/j.athoracsur.2016.03.083.

20. Narang S, Roy J, Stevens TP, Butler-O'Hara M, Mullen CA, D'Angio CT. Risk factors for umbilical venous catheter-associated thrombosis in very low birth weight infants. Pediatr Blood Cancer. 2009;52:75-9. https://doi.org/10.1002/pbc.21714.

21. Thornburg CD, Smith PB, Smithwick ML, Cotten CM, Benjamin DK Jr. Association between thrombosis and bloodstream infection in neonates with peripherally inserted catheters. Thromb Res. 2008;122:782-5. https://doi.org/ 10.1016/j.thromres.2007.10.001.

22. El-Naggar W, Yoon EW, McMillan D, Afifi J, Mitra S, Singh B, et al. Epidemiology of thrombosis in Canadian neonatal intensive care units. J Perinatol 2020;40: 1083-90. https://doi.org/10.1038/s41372-020-0678-1.

23. Children's Hospital Association-Pediatric Health Information System. PHIS. Accessed Mar 2020. https://www.childrenshospitals.org/phis.

24. Centers for Disease Control and Prevention. National Center for Health Statistics. International Classification of Diseases, (ICD-10-CM/PCS) Transition. https://www. cdc.gov/nchs/icd/icd10cm_pcs_background.htm. Accessed Aug 42021.

25. Jaffray J, Young G. Developmental hemostasis: clinical implications from the fetus to the adolescent. Pediatr Clin North Am. 2013;60:1407-17. https://doi.org/ 10.1016/j.pcl.2013.08.003

26. Society of Critical Care Medicine. 2019 Billing and Coding Changes for Peripherally Inserted Venous Catheters. Accessed Feb 2021. https://www.sccm.org/ Communications/Critical-Connections/Archives/2019/2019-Billing-and-CodingChanges-for-Peripherally-I.

27. Sharpe E, Pettit J, Ellsbury DL. A national survey of neonatal peripherally inserted central catheter (PICC) practices. Adv Neonatal Care. 2013;13:55-74. https://doi. org/10.1097/ANC.0b013e318278b907.

28. Giordano P, Grassi M, Saracco P, Molinari AC, Gentilomo C, Suppiej A, et al. Paediatric venous thromboembolism: a report from the Italian Registry of Thrombosis in Children (RITI). Blood Transfus. 2018;16:363-70. https://doi.org/ 10.2450/2017.0075-17.

29. Robinson V, Achey MA, Nag UP, Reed CR, Pahl KS, Greenberg RG, et al. Thrombosis in infants in the neonatal intensive care unit: Analysis of a large national database. J Thromb Haemost. 2021;19:400-7. https://doi.org/10.1111/jth.15144.

30. Chen $\mathrm{H}$, Zhang X, Wang $\mathrm{H}, \mathrm{Hu} \mathrm{X}$. Complications of upper extremity versus lower extremity placed peripherally inserted central catheters in neonatal intensive care units: a meta-analysis. Intensive Crit Care. Nurs. 2020:56:102753. https://doi. org/10.1016/j.iccn.2019.08.003.
31. Ma M, Garingo A, Jensen AR, Bliss D, Friedlich P. Complication risks associated with lower versus upper extremity peripherally inserted central venous catheters in neonates with gastroschisis. J Pediatr Surg. 2015;50:556-8. https://doi.org/ 10.1016/j.jpedsurg.2014.08.026.

32. Hoang V, Sills J, Chandler M, Busalani E, Clifton-Koeppel R, Modanlou HD. Percutaneously inserted central catheter for total parenteral nutrition in neonates: complications rates related to upper versus lower extremity insertion. Pediatrics. 2008;121:e1152-9. https://doi.org/10.1542/peds.2007-1962.

33. Tuckuviene R, Kristensen SR, Helgestad J, Christensen AL, Johnsen SP. Predictive value of pediatric thrombosis diagnoses in the Danish National Patient Registry. Clin Epidemiol. 2010;2:107-22. https://doi.org/10.2147/clep.s10334.

34. Setty BA, O'Brien SH, Kerlin BA. Pediatric venous thromboembolism in the United States: a tertiary care complication of chronic diseases. Pediatr Blood Cancer. 2012;59:258-64. https://doi.org/10.1002/pbc.23388.

35. Wright SB, Huskins WC, Dokholyan RS, Goldmann DA, Platt R. Administrative databases provide inaccurate data for surveillance of long-term central venous catheter-associated infections. Infect Control Hosp Epidemiol. 2003;24:946-9. https://doi.org/10.1086/502164.

\section{ACKNOWLEDGEMENTS}

The authors thank David C. Chang Ph.D., MPH, MBA for his advice regarding Stata coding.

\section{AUTHOR CONTRIBUTIONS}

The corresponding author confirms that she has had full access to the data in the study. All authors hold final responsibility for the decision to submit for publication. The following are the contributions of each author: $M C E, Y L, L Y$ CG, PF, JJ, MH and AL-conceived and/or designed the work that led to the submission, and/or acquired data, and/or played an important role in interpreting the results. MCE-drafted the paper. MCE, YL, LY, CG, PF, JJ, MH and AL-revised the paper. All authors-approved the final version and agree to be accountable for all aspects of the work.

\section{COMPETING INTERESTS}

The authors declare no competing interests.

\section{ETHICS APPROVAL AND CONSENT TO PARTICIPATE}

The Institutional Review Board of Children's Hospital Los Angeles deemed this study exempt (CHLA-20-00182).

\section{ADDITIONAL INFORMATION}

Supplementary information The online version contains supplementary material available at https://doi.org/10.1038/s41372-021-01232-1.

Correspondence and requests for materials should be addressed to Molly Crimmins Easterlin

Reprints and permission information is available at http://www.nature.com/ reprints

Publisher's note Springer Nature remains neutral with regard to jurisdictional claims in published maps and institutional affiliations. 